\title{
Fractional solutions for the inextensible Heisenberg antiferromagnetic flow and solitonic magnetic flux surfaces in the binormal direction
}

\author{
T. Körpınar ${ }^{a}$, R. Cem Demirkol ${ }^{b}$, Z. Körpınar ${ }^{c}$, and V. Asil ${ }^{d}$ \\ ${ }^{a, b}$ Muş Alparslan University, Department of Mathematics, 49250, Muş, Turkey. \\ ${ }^{c}$ Muş Alparslan University, Department of Administration, 49250, Muş, Turkey. \\ ${ }^{d}$ Firat University, Department of Mathematics, 23100, Elazı $\breve{g}$, Turkey.
}

Received 2 December 2020; accepted 20 January 2021

\begin{abstract}
Maxwellian electromagnetism describes the wave features of the light and related subjects. Its original formulation was established 150 years ago. One of the four Maxwell's equations is Gauss's law, which states significant facts regarding magnetic flux through surfaces. It was also observed that optical media provided surface electromagnetism around 60 years ago. This observation leads to improve new techniques on nano-photonics, metamaterials, and plasmonics. The goal of this manuscript is to suggest novel accurate and local conditions for defining magnetic flux surfaces for the inextensible Heisenberg antiferromagnetic flow in the binormal direction. The theoretical accuracy of the methodology is verified through the evolution of magnetic vector fields and the anti-symmetric Lorentz force field operator. On the other hand, the numerical accuracy and efficiency are developed in detail by considering the conformable fractional derivative method when these fields are transformed under the traveling wave hypothesis.
\end{abstract}

Keywords: Magnetic field lines; magnetic flux surface; geometric phase; Heisenberg antiferromagnetic flow; Lorentz force.

PACS: 03.65.Vf; 02.40.Hw; 05.45.Yv; 42.15.-i; 03.50.De.

DOI: https://doi.org/10.31349/RevMexFis.67.452

\section{Introduction}

Differential geometric tools such as surfaces and curves have been appeared in many disciplines of theoretical and practical areas of science ranging from thermodynamics [1] to high energy strings [2], and from general relativity [3] to solitons [4], or even in plasma physics [5] and liquid crystals [6]. The motion of curves and the concept of the Frenet-Serret frame are the main common ingredient in all these applications.

These tools have also been considered in the research of magnetic structures significantly. Recently, many authors have focused on the subject of magnetic curves and investigate many important results. In these studies, one common approach has been used extensively. According to this approach, it is generally assumed that magnetic curves are trajectories of the time-independent moving charged particle on geometric manifolds or physical spacetime structures. This motion of the particle is specifically determined by the Lorentz force equation. Once the Lorentz force equation is managed to solve successfully, then many interesting characterizations have been developed from the geometric and physical points of view. Körpınar and Demirkol investigated electromagnetic curves, their geometric phases, and their transportation laws, along with the linearly polarized light coupling into the optical fiber in a three-dimensional semi-Riemannian manifold [7] . Körpınar and Demirkol also obtained frictional and magnetic curves by using the antisymmetric Lorentz force operator and Frenet-Serret equations to characterize their physical and geometric properties $[8,9]$. Kazan and Karadağ computed magnetic vector fields of magnetic non-lightlike curves in terms of parallel transport frames in three-dimensional Minkowski space [10]. Güvenç and Özgür determined necessary and sufficient conditions for being slant normal magnetic curves in $(2 n+1)$-dimensional $S$-manifolds [11] . Cabrerizo studied the magnetic flow lines and associated Killing magnetic fields in three-dimensional space [12]. Sun established the connection between geometric invariants of the magnetic curves and magnetic normal binormal surfaces [13]. Körpınar et al. investigated a new kind of evolution equation for electric and magnetic fields satisfying the Maxwell equations along with the uniform optical fiber in three-dimensional ordinary space and Minkowski space $[14,15]$.

These structures are implemented by many authors to define magnetic flux-tubes in the case of inflexional configuration and inflectional disequilibrium. In the presence of a magnetic field, the magnetic flux-tube is defined by the cylindrical-thin tube of circular cross-section having a positive radius. The cases of twisted magnetic flux-tube and straight flux-tube are investigated separately in various studies. The geometric formulation of these tubes is derived by the Lorentz force equation and used to determine generic characterizations associated with the several useful applications to astrophysical flows, solar corona loops, etcetera. Nested toroidal flux surface is described due to the motion curves in magnetohydrostatic. It can be considered as a generalization of the magnetic flux-tube. All these results have been obtained through the Riemannian and non-Riemannian geometric data and facts. Ricca studied generic behavior and equilibrium conditions of the magnetic flux-tube by considering the Lorentz force equations [16]. Ricca also presented new consequences concerning inflexional and evolution in- 
stability of magnetic flux tubes when the curvature of the tube axis vanishes [17]. De Andrade obtained a non-Riemannian geometrical characterization of the magnetic flux-tube and the fluid rotation by using the Da Rios equation [18] . De Andrade showed that the twist of the magnetic flux tube could be computed in terms of the torsion of the tube axis under a special evolution equation [19]. De Andrade considered the Heisenberg spin equation and Gauss-Mainard-Codazzi equations to observe the influence of torsion and curvature of the magnetic flux-tube axis in magnetic filament acting as dynamos evolution [20] .

The wider geometric importance of the moving curves is appeared in vortex filament motion, magnetic dynamics, kinematics of interfaces. The relationship between the motion of curves and the integrable evolution equation is the main subject focused on by many researchers. As a result of this effort, the equation of the motion of curve or vortex filament is linked by many geometric and physical evolution equations such as the Landau-Lifshitz equation, non-linear Schrödinger equation, localized induction equation, Heisenberg antiferromagnetic and ferromagnetic equation, binormal equation, Da-Rios equation. Guo and Ding classified explicit or approximate boundary value solutions or initial value problems together with their physical or geometric flow models [21]. Vieria and Horley used binormal, normal, and tangent vectors of the Frenet-Serret system in such a successful way that they extracted significant data regarding the dynamics of the magnetization vector [22]. Hasimoto described an intrinsic equation governing the torsion and curvature of a vortex to define the propagation of a hump or loop of helical flow [23]. Anco and Myrzakulov generalized Heisenberg spin models and Schrödinger maps from geometric surface flows and Hamiltonian flow through Frenet-Serret equations of surfaces and curves [24] . Erdoğdu and Özdemir discussed Hasimoto surfaces and their geometric properties such as mean and Gaussian curvature of these surfaces for each case in Minkowski space [25]. Ricca realized a correlation among localized induction approximation, Betchov-Da Rios equation, and nonlinear Schrödinger equation for extracting pseudo-helicity, energy, and associated Lagrangian for the motion of a thin vortex filament [26] . Balakrishnan et al. derived the geometric phase, gauge potential, and the anholonomy density of general space curves by employing Lamb's formulation for time evolution systems [27]. Barros et al. computed soliton solutions of the Betchov-Da Rios equation explicitly in the anti-De Sitter and Lorentzian space forms $[28,29]$. Arroyo studied binormal flow with torsion and curvature to investigate the evolution of filaments [30] .

These flow models and nonlinear evolution equations can be related to soliton propagation. In many cases, they are used to explain the notion of parallel transportation and geometric phase along with that propagation. For example, the effect of this phase is observed along with the rotational direction of polarized light rays propagating through the optical fiber. If the solid angle observed by the turning of the tangent vector of optical fiber is equal to the angular rota- tion of light rays, then it implies that the polarization has been parallel transported. Subsequent studies have shown that particular equations such as Maxwell's equations, spin equations, Lorentz equations, etcetera. contain the rotational effect of the polarization of light. Köpirnar et al. studied soliton propagation of electromagnetic field vectors of the polarized light ray traveling along with the coiled optical fiber in different geometric structures [31-33]. Balakrishnan formulated path dependence of the rotation and the geometric phase of the moving orthonormal vectors by considering continuous, classical, antiferromagnetic Heisenberg flow [34]. Bliokh reviewed the spin-orbit propagation along with the light with the emphasis of the Berry phase and spin Hall effect carried by the wave [35]. Bliokh et al. also measured the precession of the Stokes vector and the spindependent deflection along the coiled ray trajectory in an excellent accuracy with theoretical estimations [36] . Wassmann and Ankiewicz improved an alternative method allowing one to derive Berry's topological phase in terms of both planar and solid angles [37] . Balakrishnan characterized a special type of geometric phase depending on a family of solitonbased equations [38]. Samuel and Nityananda explained a new transportation rule for polarization vectors and their integrability conditions while these vectors are assumed to define along with non-geodesic null curves [39] . Balakrishnan and Dandoloff generalized the classical evolution equation for a spae curve to demonstrate traditional analogs of the Heisenberg and Schrödinger pictures seen in the quantum theory [40].

This paper investigates another aspect of the moving curve evolution. We define magnetic field lines governed by the inextensible Heisenberg antiferromagnetic flow in relation to the total geometric phase and apply these to characterize the Lorentz magnetic flux surfaces in the binormal direction. Thus, we aim to derive the geometric relationship between magnetic flux surfaces and magnetic field lines in the binormal direction. The theoretical and numerical accuracy of the methodology is proved through the inextensible Heisenberg antiferromagnetic flow of magnetic vector fields, the evolution anti-symmetric Lorentz force field operator, and the conformable fractional derivative method when these fields are transformed under the traveling wave hypothesis. Thus, we aim to develop a novel approach for gaining a better insight into the nature of potential geometric and physical properties in interacting magnetic field lines and their flow models in the binormal direction.

The organization of the paper is as follows. In Sec. 2, we present differential geometric structures of orthonormal Frenet-Serret vectors and their directional derivatives. In Sec. 3, we compute magnetic and electric $b$-lines in terms of orthonormal vector fields and associated geometric quantities in the binormal direction. In Sec. 4, we describe a new type of inextensible Heisenberg antiferromagnetic flow model for the magnetic lines and associated magnetic vector fields in the binormal direction. In Sec. 5, the results and potential research topics are specialized. We conclude the paper by 
the appendix, which includes approximate solutions of some equations computed through the manuscript.

\section{Differential Geometry of Frenet-Serret Vec- tors}

In the introduction, we state that the motion of curves and the concept of the Frenet-Serret frame are the main common ingredient in many applications. The orthonormal FrenetSerret frame governing the intrinsic characterization of the vector triple $\mathcal{A}=\left(\overrightarrow{\mathbf{e}_{1}}, \overrightarrow{\mathbf{e}_{2}}, \overrightarrow{\mathbf{e}_{3}}\right)$ is given in the compact shape by

$$
\begin{aligned}
\frac{\partial}{\partial s} \mathcal{A}^{T} & =\mathcal{X} \mathcal{A}^{T}, \\
\frac{\partial}{\partial n} \mathcal{A}^{T} & =\mathcal{Y} \mathcal{A}^{T}, \\
\frac{\partial}{\partial b} \mathcal{A}^{T} & =\mathcal{Z} \mathcal{A}^{T},
\end{aligned}
$$

where $\mathcal{X}, \mathcal{Y}, \mathcal{Z}$ can be written in the following skewsymmetric matrix form

$$
\begin{aligned}
& \mathcal{X}=\left(\begin{array}{ccc}
0 & \kappa_{1} & 0 \\
-\kappa_{1} & 0 & \kappa_{2} \\
0 & -\kappa_{2} & 0
\end{array}\right), \\
& \mathcal{Y}=\left(\begin{array}{ccc}
0 & \kappa_{n s} & \kappa_{b}+\kappa_{2} \\
-\kappa_{n s} & 0 & -\operatorname{div} \overrightarrow{\mathbf{e}_{3}} \\
-\left(\kappa_{b}+\kappa_{2}\right) & \operatorname{div} \overrightarrow{\mathbf{e}_{3}} & 0
\end{array}\right), \\
& \mathcal{Z}=\left(\begin{array}{ccc}
0 & -\left(\kappa_{n}+\kappa_{2}\right) & \kappa_{b s} \\
\left(\kappa_{n}+\kappa_{2}\right) & 0 & \kappa_{1}+\operatorname{div} \overrightarrow{\mathbf{e}_{2}} \\
-\kappa_{b s} & -\left(\kappa_{1}+\operatorname{div} \overrightarrow{\mathbf{e}_{2}}\right) & 0
\end{array}\right)
\end{aligned}
$$

respectively. Here, $T$ denotes the transpose of the matrix $\mathcal{A}=\left(\overrightarrow{\mathbf{e}_{1}}, \overrightarrow{\mathbf{e}_{2}}, \overrightarrow{\mathbf{e}_{3}}\right)$. Furthermore, $\partial / \partial s, \partial / \partial n, \partial / \partial b$ represent directional derivatives in the tangent direction, normal direction, and binormal direction, respectively. By assumption, in the tangent direction, we know that the tangent vector of $s$-lines is denoted by $\overrightarrow{\mathbf{e}_{1}}$ i.e.

$$
\frac{\partial}{\partial s} \varsigma=\overrightarrow{\mathbf{e}_{1}}
$$

where $\varsigma$ is assumed to represent $s$-lines. In the normal direction, the tangent vector of $n$-lines is denoted by $\overrightarrow{\mathbf{e}_{2}}$ i.e.

$$
\frac{\partial}{\partial n} \varpi=\overrightarrow{\mathbf{e}_{2}}
$$

where $\varpi$ is assumed to represent $n$-lines. In the binormal direction, the tangent vector of $b$-lines is denoted by $\overrightarrow{\mathbf{e}_{3}}$ i.e.

$$
\frac{\partial}{\partial b} \theta=\overrightarrow{\mathbf{e}_{3}}
$$

where $\theta$ is assumed to represent $b$-lines. The gradient operator is written by

$$
\nabla=\overrightarrow{\mathbf{e}_{1}} \frac{\partial}{\partial s}+\overrightarrow{\mathbf{e}_{2}} \frac{\partial}{\partial n}+\overrightarrow{\mathbf{e}_{3}} \frac{\partial}{\partial b}
$$

Hence, the vector analysis formulas can be expressed by

$$
\begin{aligned}
& \operatorname{div} \overrightarrow{\mathbf{e}_{1}}=\kappa_{n s}+\kappa_{b s}, \\
& \operatorname{div} \overrightarrow{\mathbf{e}_{2}}=-\kappa_{1}+\overrightarrow{\mathbf{e}_{3}} \frac{\partial}{\partial b} \overrightarrow{\mathbf{e}_{2}}, \\
& \operatorname{div} \overrightarrow{\mathbf{e}_{3}}=-\overrightarrow{\mathbf{e}_{3}} \frac{\partial}{\partial n} \overrightarrow{\mathbf{e}_{2}},
\end{aligned}
$$

where

$$
\kappa_{n s}=\overrightarrow{\mathbf{e}_{2}} \frac{\partial}{\partial n} \overrightarrow{\mathbf{e}_{1}}, \kappa_{b s}=\overrightarrow{\mathbf{e}_{3}} \frac{\partial}{\partial b} \overrightarrow{\mathbf{e}_{1}}
$$

The curl operator is written by

$$
\nabla=\overrightarrow{\mathbf{e}_{1}} \times \frac{\partial}{\partial s}+\overrightarrow{\mathbf{e}_{2}} \times \frac{\partial}{\partial n}+\overrightarrow{\mathbf{e}_{3}} \times \frac{\partial}{\partial b}
$$

Hence, the other vector analysis formulas can be expressed by

$$
\begin{aligned}
& \operatorname{curl} \overrightarrow{\mathbf{e}_{1}}=\kappa_{s} \overrightarrow{\mathbf{e}_{1}}+\kappa_{1} \overrightarrow{\mathbf{e}_{3}}, \\
& \operatorname{curl} \overrightarrow{\mathbf{e}_{2}}=-\left(\operatorname{div} \overrightarrow{\mathbf{e}_{3}}\right) \overrightarrow{\mathbf{e}_{1}}+\kappa_{n} \overrightarrow{\mathbf{e}_{2}}+\kappa_{n s} \overrightarrow{\mathbf{e}_{3}}, \\
& \operatorname{curl} \overrightarrow{\mathbf{e}_{3}}=\left(\kappa_{1}+\operatorname{div} \mathbf{e}_{2}\right) \overrightarrow{\mathbf{e}_{1}}-\kappa_{b s} \overrightarrow{\mathbf{e}_{2}}+\kappa_{b} \overrightarrow{\mathbf{e}_{3}},
\end{aligned}
$$

where

$$
\begin{aligned}
\kappa_{s} & =\overrightarrow{\mathbf{e}_{3}} \frac{\partial}{\partial n} \overrightarrow{\mathbf{e}_{1}}-\overrightarrow{\mathbf{e}_{2}} \frac{\partial}{\partial b} \overrightarrow{\mathbf{e}_{1}} \\
\kappa_{n} & =\overrightarrow{\mathbf{e}_{1}} \frac{\partial}{\partial b} \overrightarrow{\mathbf{e}_{2}}-\kappa_{2} \\
\kappa_{b} & =-\kappa_{2}-\overrightarrow{\mathbf{e}_{1}} \frac{\partial}{\partial n} \overrightarrow{\mathbf{e}_{3}}
\end{aligned}
$$

In the end, we remind that each vector or geometric quantity depends on the three variables of $(s, n, b)$. For the brevity purpose, we rather choose using $\overrightarrow{\mathbf{e}_{1}}=\overrightarrow{\mathbf{e}_{1}}(s, n, b), \kappa_{1}=$ $\kappa_{1}(s, n, b)$, etc. type of notation. We will continue to apply a similar notation for any function associated with the above vectors or geometric quantities. We also think that repeated statements of smoothness conditions and excessive repetition that certain parameters are supposed to be non-vanishing may seem obscure. Accordingly, we suppose that all functions are sufficiently smooth as required by the calculation unless stated otherwise.

\section{Magnetic and electric $b$-lines in the binor- mal direction}

An important relation between electromagnetism and differential geometry can be established when the moving positively charged particle under the action of the Lorentz force and its trajectory is observed. This trajectory is represented by electromagnetic lines such that they are assumed to the composition of magnetic lines and electric lines. Lorentz force has a crucial role to describe the behavior of these lines. 
In the differential geometric viewpoint, the magnetic $s$-lines, magnetic $n$-lines, magnetic $b$-lines satisfy the following types of Lorentz force equations in the tangent direction, normal direction, and binormal direction, respectively

$$
\begin{aligned}
& \phi_{s}\left(\overrightarrow{\mathbf{e}_{1}}\right)=\frac{\partial}{\partial s} \overrightarrow{\mathbf{e}_{1}}=\mathcal{M}_{s} \times \overrightarrow{\mathbf{e}_{1}} \\
& \phi_{n}\left(\overrightarrow{\mathbf{e}_{2}}\right)=\frac{\partial}{\partial n} \overrightarrow{\mathbf{e}_{2}}=\mathcal{M}_{n} \times \overrightarrow{\mathbf{e}_{2}} \\
& \phi_{b}\left(\overrightarrow{\mathbf{e}_{3}}\right)=\frac{\partial}{\partial b} \overrightarrow{\mathbf{e}_{3}}=\mathcal{M}_{b} \times \overrightarrow{\mathbf{e}_{3}},
\end{aligned}
$$

where $\mathcal{M}_{s}, \mathcal{M}_{n}, \mathcal{M}_{b}$ are divergenceless magnetic vector fields associated with each magnetic line given directions [41]. By using the language of differential geometry, we aim to simplify the calculation and formalization of the magnetic field lines and provide various other physical understanding more simply. In this section, we obtain magnetic lines under the action of the Lorentz force equation by using the FrenetSerret formalism in the binormal direction. Later on, these lines will be used derived magnetic flux surfaces when their motions are governed by the inextensible Heisenberg antiferromagnetic flow.

First, we consider Eqs. $(5,23)$ to obtain Lorentz force fields of Frenet-Serret vectors. Then we should also take into account the following well-known facts of the inner product

$$
\begin{aligned}
\phi_{b}\left(\overrightarrow{\mathbf{e}_{1}}\right) \cdot \overrightarrow{\mathbf{e}_{2}} & =-\phi_{b}\left(\overrightarrow{\mathbf{e}_{2}}\right) \cdot \overrightarrow{\mathbf{e}_{1}} \phi_{b}\left(\overrightarrow{\mathbf{e}_{1}}\right) \cdot \overrightarrow{\mathbf{e}_{3}} \\
& =-\phi_{b}\left(\overrightarrow{\mathbf{e}_{3}}\right) \cdot \overrightarrow{\mathbf{e}_{1}}, \phi_{b}\left(\overrightarrow{\mathbf{e}_{2}}\right) \cdot \overrightarrow{\mathbf{e}_{3}}=-\phi_{b}\left(\overrightarrow{\mathbf{e}_{3}}\right) \cdot \overrightarrow{\mathbf{e}_{2}} .
\end{aligned}
$$

So, from Eqs. $(5,23,24)$, Lorentz force fields of Frenet-Serret vectors are obtained in the following way

$$
\begin{aligned}
\phi_{b}\left(\overrightarrow{\mathbf{e}_{1}}\right) & =\rho \overrightarrow{\mathbf{e}_{2}}+\kappa_{b s} \overrightarrow{\mathbf{e}_{3}}, \phi_{b}\left(\overrightarrow{\mathbf{e}_{2}}\right) \\
& =-\rho \overrightarrow{\mathbf{e}_{1}}+\left(\kappa_{1}+\operatorname{div} \overrightarrow{\mathbf{e}_{2}}\right) \overrightarrow{\mathbf{e}_{3}}, \phi_{b}\left(\overrightarrow{\mathbf{e}_{3}}\right) \\
& =-\kappa_{b s} \overrightarrow{\mathbf{e}_{1}}-\left(\kappa_{1}+\operatorname{div} \overrightarrow{\mathbf{e}_{2}}\right) \overrightarrow{\mathbf{e}_{2}},
\end{aligned}
$$

where $\rho$ is a well-defined arbitrarily chosen sufficiently smooth function along with magnetic $b$-lines. It is already known that divergenceless magnetic vector field $\mathcal{M}_{b}$ is spanned by the $\left(\overrightarrow{\mathbf{e}_{1}}, \overrightarrow{\mathbf{e}_{2}}, \overrightarrow{\mathbf{e}_{3}}\right)$. Therefore, it should also be true that

$$
\mathcal{M}_{b}=m_{1} \overrightarrow{\mathbf{e}_{1}}+m_{2} \overrightarrow{\mathbf{e}_{2}}+m_{3} \overrightarrow{\mathbf{e}_{3}}
$$

where $\phi_{b}\left(\mathcal{M}_{b}\right)=0$. Here, $m_{1}, m_{2}$, and $m_{3}$ are also sufficiently smooth functions along with magnetic $b$-lines. As a result of Eqs. $(23,25,26,27)$, the magnetic vector field of magnetic $b$-lines in the binormal direction is computed by

$$
\mathcal{M}_{b}=\left(\kappa_{1}+\operatorname{div} \overrightarrow{\mathbf{e}_{2}}\right) \overrightarrow{\mathbf{e}_{1}}-\kappa_{b s} \overrightarrow{\mathbf{e}_{2}}+\rho \overrightarrow{\mathbf{e}_{3}}
$$

The electromagnetic force equation in the binormal direction can be expressed by

$$
\mathcal{F}_{b}=q\left(\mathcal{E}_{b}+\overrightarrow{\mathbf{e}_{3}} \times \mathcal{M}_{b}\right)=m \phi_{b}\left(\overrightarrow{\mathbf{e}_{3}}\right),
$$

where $\mathcal{E}_{n}$ denotes electric $n$-lines defined along with the motion of the positively charged particle. Thus, by using Eqs. $(28,29)$, it is obtained that

$$
\mathcal{E}_{b}=-\kappa_{b s}(1+m / q) \overrightarrow{\mathbf{e}_{1}}-\left(\kappa_{1}+\operatorname{div} \overrightarrow{\mathbf{e}_{2}}\right)(1+m / q) \overrightarrow{\mathbf{e}_{2}}
$$

\section{Inextensible Heisenberg antiferromagnetic flow model in the binormal direction}

The inextensibility of the mechanism is an important tool to seek the relation between the geometric motion of space or plane curves and integrable equations. Apart from the traditional inextensible flows in the tangent direction, we identify time-dependent evolution equations satisfied by the geometric quantities of magnetic $b$-lines under inextensible flows in the binormal direction.

In the binormal direction, the most generalized form of the inextensible flow can be represented by

$$
\frac{\partial}{\partial u} \varpi=\lambda_{1} \overrightarrow{\mathbf{e}_{1}}+\lambda_{2} \overrightarrow{\mathbf{e}_{2}}+\lambda_{3} \overrightarrow{\mathbf{e}_{3}}
$$

where $\lambda_{1}, \lambda_{2}$, and $\lambda_{3}$ are sufficiently smooth coefficients of the tangent, normal, and binormal vectors along magnetic $b$ lines in the binormal direction. $\partial / \partial u$ is used to represent the time derivative. Here, one should also recall that the binormal vector is assumed to satisfy the following identity

$$
\frac{\partial}{\partial b} \varpi=\overrightarrow{\mathbf{e}_{3}}
$$

where $\varpi$ denotes the family of magnetic $b$-lines in the binormal direction. Now, if basic compatibility conditions and properties of the inner product are applied to Eqs. $(5,31,32)$ then we may write that

$$
\begin{aligned}
\frac{\partial}{\partial u} \overrightarrow{\mathbf{e}_{1}} & =\sigma \overrightarrow{\mathbf{e}_{2}}-\left(\frac{\partial}{\partial b} \lambda_{1}+\lambda_{2}\left(\kappa_{n}+\kappa_{2}\right)-\lambda_{3} \kappa_{b s}\right) \overrightarrow{\mathbf{e}_{3}} \\
\frac{\partial}{\partial u} \overrightarrow{\mathbf{e}_{2}} & =-\sigma \overrightarrow{\mathbf{e}_{1}} \\
& -\left(\frac{\partial}{\partial b} \lambda_{2}-\lambda_{1}\left(\kappa_{n}+\kappa_{2}\right)-\lambda_{3}\left(\kappa_{1}+d i v \overrightarrow{\mathbf{e}_{2}}\right)\right) \overrightarrow{\mathbf{e}_{3}} \\
\frac{\partial}{\partial u} \overrightarrow{\mathbf{e}_{3}} & =\left(\frac{\partial}{\partial b} \lambda_{1}+\lambda_{2}\left(\kappa_{n}+\kappa_{2}\right)-\lambda_{3} \kappa_{b s}\right) \overrightarrow{\mathbf{e}_{1}} \\
& +\left(\frac{\partial}{\partial b} \lambda_{2}-\lambda_{1}\left(\kappa_{n}+\kappa_{2}\right)-\lambda_{3}\left(\kappa_{1}+d i v \overrightarrow{\mathbf{e}_{2}}\right)\right) \overrightarrow{\mathbf{e}_{2}}
\end{aligned}
$$

where $\sigma$ is a sufficiently smooth function defined along with the magnetic $b$-lines in the binormal direction. Eqs. (32-34) are given the most generalized form of the evolution equation for time derivative in the normal direction. Now, we define the particular case of the inextensible evolution of magnetic $b$-lines in the binormal direction. This particular case is known as the Heisenberg antiferromagnetic flow in the binormal direction. It is defined by

$$
\frac{\partial}{\partial u} \varpi=\frac{\partial}{\partial b} \varpi \times \frac{\partial^{2}}{\partial b^{2}} \varpi,
$$


or equivalently

$$
\frac{\partial}{\partial u} \varpi=\overrightarrow{\mathbf{e}_{3}} \times \phi_{b}\left(\overrightarrow{\mathbf{e}_{3}}\right)
$$

From Eqs. $(5,35,36)$, it can easily be computed that

$$
\frac{\partial}{\partial u} \varpi=\left(\kappa_{1}+\operatorname{div} \overrightarrow{\mathbf{e}_{2}}\right) \overrightarrow{\mathbf{e}_{1}}-\left(\kappa_{b s}\right) \overrightarrow{\mathbf{e}_{2}}
$$

By comparing Eqs. (32-34) and Eq. (37), we can conclude that the inextensible flow and Heisenberg antiferromagnetic flow of $b$-magnetic lines coincide in the binormal direction when the following equalities satisfy

$$
\lambda_{1}=\kappa_{1}+\operatorname{div} \overrightarrow{\mathbf{e}_{2}}, \lambda_{2}=-\kappa_{b s}, \lambda_{3}=0
$$

Thus, the inextensible Heisenberg antiferromagnetic flow of Frenet-Serret vectors can be induced to

$$
\begin{aligned}
& \frac{\partial}{\partial u} \overrightarrow{\mathbf{e}_{1}}=\sigma \overrightarrow{\mathbf{e}_{2}}-\left(\frac{\partial}{\partial b}\left(\kappa_{1}+\operatorname{div} \overrightarrow{\mathbf{e}_{2}}\right)-\kappa_{b s}\left(\kappa_{n}+\kappa_{2}\right)\right) \overrightarrow{\mathbf{e}_{3}}, \\
& \frac{\partial}{\partial u} \overrightarrow{\mathbf{e}_{2}}=-\sigma \overrightarrow{\mathbf{e}_{1}}+\left(\frac{\partial}{\partial b} \kappa_{b s}+\left(\kappa_{1}+\operatorname{div} \overrightarrow{\overrightarrow{\mathbf{e}_{2}}}\right)\left(\kappa_{n}+\kappa_{2}\right)\right) \overrightarrow{\mathbf{e}_{3}}, \\
& \frac{\partial}{\partial u} \overrightarrow{\mathbf{e}_{3}}=\left(\frac{\partial}{\partial b}\left(\kappa_{1}+d i v \overrightarrow{\mathbf{e}_{2}}\right)-\kappa_{b s}\left(\kappa_{n}+\kappa_{2}\right)\right) \overrightarrow{\mathbf{e}_{1}}-\left(\frac{\partial}{\partial b} \kappa_{b s}+\left(\kappa_{1}+d i v \overrightarrow{\mathbf{e}_{2}}\right)\left(\kappa_{n}+\kappa_{2}\right)\right) \overrightarrow{\mathbf{e}_{2}},
\end{aligned}
$$

where $\sigma$ is a sufficiently smooth function defined along with the magnetic $b$-lines in the binormal direction. Time-dependent evolution equations of Frenet-Serret vectors may also be written by only using the vector calculus identities in the following way

$$
\begin{aligned}
& \frac{\partial}{\partial u} \overrightarrow{\mathbf{e}_{1}}=\sigma \overrightarrow{\mathbf{e}_{2}}-\left(\frac{\partial}{\partial b}\left[\overrightarrow{\mathbf{e}_{3}} \frac{\partial}{\partial b} \overrightarrow{\mathbf{e}_{2}}\right]-\overrightarrow{\mathbf{e}_{3}} \frac{\partial}{\partial b} \overrightarrow{\mathbf{e}_{1}}\left[\overrightarrow{\mathbf{e}_{1}} \frac{\partial}{\partial b} \overrightarrow{\mathbf{e}_{2}}\right]\right) \overrightarrow{\mathbf{e}_{3}} \\
& \frac{\partial}{\partial u} \overrightarrow{\mathbf{e}_{2}}=-\sigma \overrightarrow{\mathbf{e}_{1}}+\left(\frac{\partial}{\partial b}\left[\overrightarrow{\mathbf{e}_{3}} \frac{\partial}{\partial b} \overrightarrow{\mathbf{e}_{1}}\right]+\overrightarrow{\mathbf{e}_{3}} \frac{\partial}{\partial b} \overrightarrow{\mathbf{e}_{2}}\left[\overrightarrow{\mathbf{e}_{1}} \frac{\partial}{\partial b} \overrightarrow{\mathbf{e}_{2}}\right]\right) \overrightarrow{\mathbf{e}_{3}} \\
& \frac{\partial}{\partial u} \overrightarrow{\mathbf{e}_{3}}=\left(\frac{\partial}{\partial b}\left[\overrightarrow{\mathbf{e}_{3}} \frac{\partial}{\partial b} \overrightarrow{\mathbf{e}_{2}}\right]-\overrightarrow{\mathbf{e}_{3}} \frac{\partial}{\partial b} \overrightarrow{\mathbf{e}_{1}}\left[\overrightarrow{\mathbf{e}_{1}} \frac{\partial}{\partial b} \overrightarrow{\mathbf{e}_{2}}\right]\right) \overrightarrow{\mathbf{e}_{1}}-\left(\frac{\partial}{\partial b}\left[\overrightarrow{\mathbf{e}_{3}} \frac{\partial}{\partial b} \overrightarrow{\mathbf{e}_{1}}\right]+\overrightarrow{\mathbf{e}_{3}} \frac{\partial}{\partial b} \overrightarrow{\mathbf{e}_{2}}\left[\overrightarrow{\mathbf{e}_{1}} \frac{\partial}{\partial b} \overrightarrow{\mathbf{e}_{2}}\right]\right) \overrightarrow{\mathbf{e}_{2}}
\end{aligned}
$$

The inextensible soliton surface associated with the Heisenberg antiferromagnetic flow of magnetic $b$-lines can be constructed once the coefficients of the fundamental forms are derived. Coefficients of the first fundamental form can be computed by

$$
\begin{aligned}
\mathcal{I} & =(d \varpi \cdot d \varpi)=\left(\frac{\partial}{\partial b} \varpi d b+\frac{\partial}{\partial u} \varpi d u \cdot \frac{\partial}{\partial b} \varpi d b+\frac{\partial}{\partial u} \varpi d u\right)=\left(\overrightarrow{\mathbf{e}_{3}} d b+\left(\kappa_{1}+d i v \overrightarrow{\mathbf{e}_{2}}\left(\overrightarrow{\mathbf{e}_{1}} d u-\kappa_{b s} \overrightarrow{\mathbf{e}_{2}} d u \cdot \overrightarrow{\mathbf{e}_{3}} d b\right.\right.\right. \\
& \left.+\left(\kappa_{1}+d i v \overrightarrow{\mathbf{e}_{2}}\right) \overrightarrow{\mathbf{e}_{1}} d u-\kappa_{b s} \overrightarrow{\mathbf{e}_{2}} d u\right)=d b^{2}+\left(\left(\kappa_{1}+d i v \overrightarrow{\mathbf{e}_{2}}\right)^{2}+\left(\kappa_{b s}\right)^{2}\right) d u^{2} .
\end{aligned}
$$

Thus, we find that

$$
\mathcal{E}_{\mathcal{I}}=1, \mathcal{F}_{\mathcal{I}}=0, \mathcal{G}_{\mathcal{I}}=\left(\kappa_{1}+\operatorname{div} \overrightarrow{\mathbf{e}_{2}}\right)^{2}+\left(\kappa_{b s}\right)^{2}
$$

The binormal vector of the inextensible soliton surface associated with the Heisenberg antiferromagnetic flow of magnetic $b$-lines is calculated as

$$
\mathcal{N}=\frac{\frac{\partial}{\partial b} \varpi \times \frac{\partial}{\partial u} \varpi}{\left\|\frac{\partial}{\partial b} \varpi \times \frac{\partial}{\partial u} \varpi\right\|}=\frac{\kappa_{b s} \overrightarrow{\mathbf{e}_{1}}+\left(\kappa_{1}+\operatorname{div} \overrightarrow{\mathbf{e}_{2}}\right) \overrightarrow{\mathbf{e}_{2}}}{\sqrt{\left(\kappa_{b s}\right)^{2}+\left(\kappa_{1}+d i v \overrightarrow{\mathbf{e}_{2}}\right)^{2}}}
$$


Then, coefficients of the second fundamental form can be computed by

$$
\begin{aligned}
\mathcal{I I} & =(d \varpi \cdot d \mathcal{N})=\left(\frac{\partial}{\partial b} \varpi d b+\frac{\partial}{\partial u} \varpi d u \cdot \frac{\partial}{\partial b} \mathcal{N} d b+\frac{\partial}{\partial u} \mathcal{N} d u\right)=\frac{1}{\sqrt{\left(\kappa_{b s}\right)^{2}+\left(\kappa_{1}+d i v \overrightarrow{\mathbf{e}_{2}}\right)^{2}}}\left(\overrightarrow{\mathbf{e}_{3}} d b+\left[\kappa_{1}+d i v \overrightarrow{\mathbf{e}_{2}}\right] \overrightarrow{\mathbf{e}_{1}} d u\right. \\
& -\kappa_{b s} \overrightarrow{\mathbf{e}_{2}} d u \cdot\left[\frac{\partial}{\partial b} \kappa_{b s}+\left\{\kappa_{1}+d i v \overrightarrow{\mathbf{e}_{2}}\right\}\left\{\kappa_{n}+\kappa_{2}\right\}\right] \overrightarrow{\mathbf{e}_{1}} d b+\left[\frac{\partial}{\partial b}\left\{\kappa_{1}+d i v \overrightarrow{\mathbf{e}_{2}}\right\}-\kappa_{b s}\left\{\kappa_{n}+\kappa_{2}\right\}\right] \overrightarrow{\mathbf{e}_{2}} d b+\left[\left\{\kappa_{b s}\right\}^{2}\right. \\
& \left.+\left\{\kappa_{1}+d i v \overrightarrow{\mathbf{e}_{2}}\right\}^{2}\right] \overrightarrow{\mathbf{e}_{3}} d b+\left[\frac{\partial}{\partial u} \kappa_{b s}-\left\{\kappa_{1}+d i v \overrightarrow{\mathbf{e}_{2}}\right\} \sigma\right] \overrightarrow{\mathbf{e}_{1}} d u+\left[\frac{\partial}{\partial u}\left\{\kappa_{1}+d i v \overrightarrow{\mathbf{e}_{2}}\right\}+\kappa_{b s} \sigma\right] \overrightarrow{\mathbf{e}_{2}} d u+\left[\left\{\kappa_{b s}\right\}^{2}\right. \\
& \left.\left.+\left\{\kappa_{1}+d i v \overrightarrow{\mathbf{e}_{2}}\right\}^{2}\left\{\kappa_{n}+\kappa_{2}\right\}-\kappa_{b s} \frac{\partial}{\partial b}\left\{\kappa_{1}+d i v \overrightarrow{\mathbf{e}_{2}}\right\}+\left\{\kappa_{1}+d i v \overrightarrow{\mathbf{e}_{2}}\right\} \frac{\partial}{\partial b} \kappa_{b s}\right]\right) \overrightarrow{\mathbf{e}_{3}} d u .
\end{aligned}
$$

A direct computation of the above equation yields that

$$
\begin{aligned}
\mathcal{I I} & =\frac{1}{\sqrt{\left(\kappa_{b s}\right)^{2}+\left(\kappa_{1}+\operatorname{div} \overrightarrow{\mathbf{e}_{2}}\right)^{2}}}\left(\left[\left\{\kappa_{b s}\right\}^{2}+\left\{\kappa_{1}+\operatorname{div} \overrightarrow{\mathbf{e}_{2}}\right\}^{2}\right] d b^{2}+2\left[\left\{\left(\kappa_{b s}\right)^{2}+\left(\kappa_{1}+\operatorname{div} \overrightarrow{\mathbf{e}_{2}}\right)^{2}\right\}\left\{\kappa_{n}+\kappa_{2}\right\}-\kappa_{b s} \frac{\partial}{\partial b}\left\{\kappa_{1}+\operatorname{div} \overrightarrow{\mathbf{e}_{2}}\right\}\right.\right. \\
& \left.\left.+\left\{\kappa_{1}+\operatorname{div} \overrightarrow{\mathbf{e}_{2}}\right\} \frac{\partial}{\partial b} \kappa_{b s}\right] d b d u+\left[-\sigma\left\{\left(\kappa_{b s}\right)^{2}+\left(\kappa_{1}+\operatorname{div} \overrightarrow{\mathbf{e}_{2}}\right)^{2}\right\}-\kappa_{b s} \frac{\partial}{\partial u}\left\{\kappa_{1}+\operatorname{div} \overrightarrow{\mathbf{e}_{2}}\right\}+\left\{\kappa_{1}+\operatorname{div} \overrightarrow{\mathbf{e}_{2}}\right\} \frac{\partial}{\partial u} \kappa_{b s}\right] d u^{2}\right) .
\end{aligned}
$$

Thus, we find that

$$
\begin{aligned}
& \mathcal{E}_{\mathcal{I I}}=\frac{1}{\sqrt{\left(\kappa_{b s}\right)^{2}+\left(\kappa_{1}+\operatorname{div} \overrightarrow{\mathbf{e}_{2}}\right)^{2}}}\left(\left[\kappa_{b s}\right]^{2}+\left[\kappa_{1}+\operatorname{div} \overrightarrow{\mathbf{e}_{2}}\right]^{2}\right), \\
& \mathcal{F}_{\mathcal{I I}}=\frac{2}{\sqrt{\left(\kappa_{b s}\right)^{2}+\left(\kappa_{1}+\operatorname{div} \overrightarrow{\mathbf{e}_{2}}\right)^{2}}}\left(\left[\left\{\kappa_{b s}\right\}^{2}+\left\{\kappa_{1}+\operatorname{div} \overrightarrow{\mathbf{e}_{2}}\right\}^{2}\right]\left[\kappa_{n}+\kappa_{2}\right]-\kappa_{b s} \frac{\partial}{\partial b}\left[\kappa_{1}+\operatorname{div} \overrightarrow{\mathbf{e}_{2}}\right]+\left[\kappa_{1}+\operatorname{div} \overrightarrow{\mathbf{e}_{2}}\right] \frac{\partial}{\partial b} \kappa_{b s}\right), \\
& \mathcal{G}_{\mathcal{I I}}=\frac{1}{\sqrt{\left(\kappa_{b s}\right)^{2}+\left(\kappa_{1}+\operatorname{div} \overrightarrow{\mathbf{e}_{2}}\right)^{2}}}\left(-\sigma\left[\left\{\kappa_{b s}\right\}^{2}+\left\{\kappa_{1}+\operatorname{div} \overrightarrow{\mathbf{e}_{2}}\right\}^{2}\right]-\kappa_{b s} \frac{\partial}{\partial u}\left[\kappa_{1}+\operatorname{div} \overrightarrow{\mathbf{e}_{2}}\right]+\left[\kappa_{1}+\operatorname{div} \overrightarrow{\mathbf{e}_{2}}\right] \frac{\partial}{\partial u} \kappa_{b s}\right) .
\end{aligned}
$$

As a result, the Gauss curvature and mean curvature of the inextensible soliton surface associated with the Heisenberg antiferromagnetic flow of magnetic $b$-lines are given by using Eqs. (45-52) in the following way

$$
\begin{aligned}
\mathcal{G}_{\mathcal{C}} & =\frac{1}{\left(\kappa_{b s}\right)^{2}+\left(\kappa_{1}+\operatorname{div} \overrightarrow{\mathbf{e}_{2}}\right)^{2}}\left(-\sigma\left[\left\{\kappa_{b s}\right\}^{2}+\left\{\kappa_{1}+\operatorname{div} \overrightarrow{\mathbf{e}_{2}}\right\}^{2}\right]-\kappa_{b s} \frac{\partial}{\partial u}\left[\kappa_{1}+\operatorname{div} \overrightarrow{\mathbf{e}_{2}}\right]+\left[\kappa_{1}+\operatorname{div} \overrightarrow{\mathbf{e}_{2}}\right] \frac{\partial}{\partial u} \kappa_{b s}\right) \\
& -\frac{4}{\left(\left(\kappa_{b s}\right)^{2}+\left(\kappa_{1}+\operatorname{div} \overrightarrow{\mathbf{e}_{2}}\right)^{2}\right)^{2}}\left(\left[\left\{\kappa_{b s}\right\}^{2}+\left\{\kappa_{1}+\operatorname{div} \overrightarrow{\mathbf{e}_{2}}\right\}^{2}\right]\left[\kappa_{n}+\kappa_{2}\right]-\kappa_{b s} \frac{\partial}{\partial b}\left[\kappa_{1}+\operatorname{div} \overrightarrow{\mathbf{e}_{2}}\right]+\left[\kappa_{1}+\operatorname{div} \overrightarrow{\mathbf{e}_{2}}\right] \frac{\partial}{\partial b} \kappa_{b s}\right) \\
\mathcal{H}_{\mathcal{C}} & =\frac{1}{2\left(\left(\kappa_{b s}\right)^{2}+\left(\kappa_{1}+\operatorname{div} \overrightarrow{\mathbf{e}_{2}}\right)^{2}\right)^{3 / 2}}\left(\left[-\sigma+\left\{\kappa_{b s}\right\}^{2}+\left\{\kappa_{1}+\operatorname{div} \overrightarrow{\mathbf{e}_{2}}\right\}^{2}\right]\left[\left\{\kappa_{b s}\right\}^{2}+\left\{\kappa_{1}+\operatorname{div} \overrightarrow{\mathbf{e}_{2}}\right\}^{2}\right]\right. \\
& \left.+\left[\kappa_{1}+\operatorname{div} \overrightarrow{\mathbf{e}_{2}}\right] \frac{\partial}{\partial u} \kappa_{b s}-\kappa_{b s} \frac{\partial}{\partial u}\left[\kappa_{1}+\operatorname{div} \overrightarrow{\mathbf{e}_{2}}\right]\right)
\end{aligned}
$$

Now, we can present some further geometric properties of magnetic $b$-lines lying on the inextensible soliton surface associated with the Heisenberg antiferromagnetic flow in the binormal direction.

A curve lying on any surface is called a geodesic if and only if the normal vector of the surface coincides with the principal normal of the curve. Based on this fact, we get the following two results.

$b$-parameter magnetic $b$-lines of the inextensible soliton surface associated with the Heisenberg antiferromagnetic flow in the binormal direction are geodesics.

$\checkmark u$-parameter magnetic $b$-lines of the inextensible soliton surface associated with the Heisenberg antiferromagnetic flow in the binormal direction are geodesics if and only if

$$
\kappa_{b s} \frac{\partial}{\partial b} \kappa_{b s}+\left(\kappa_{1}+\operatorname{div} \overrightarrow{\mathbf{e}_{2}}\right) \frac{\partial}{\partial b}\left(\kappa_{1}+\operatorname{div} \overrightarrow{\mathbf{e}_{2}}\right)=0 .
$$


A curve lying on any surface is called an asymptotic if and only if along the curve the surface normal vector field is orthogonal to the principal normal vector field of the curve. Based on this fact, we get the following two results.

- $b$-parameter magnetic $b$-lines of the inextensible soliton surface associated with the Heisenberg antiferromagnetic flow in the binormal direction are asymptotics if and only if

$$
\kappa_{1}=-\operatorname{div} \overrightarrow{\mathbf{e}_{2}}
$$

$u$-parameter magnetic $b$-lines of the inextensible soliton surface associated with the Heisenberg antiferromagnetic flow in the binormal direction are asymptotics if and only if

$$
\kappa_{b s}=\sigma\left(\kappa_{1}+d i v \overrightarrow{\mathbf{e}_{2}}\right)
$$

As a result, we conclude that magnetic $b$-lines are lines of curvature if and only if the following equality holds

$$
-\left(\kappa_{1}+\operatorname{div} \overrightarrow{\mathbf{e}_{2}}\right) \frac{\partial}{\partial b} \kappa_{b s}+\kappa_{b s} \frac{\partial}{\partial b}\left(\kappa_{1}+\operatorname{div} \overrightarrow{\mathbf{e}_{2}}\right)=\left(\kappa_{n}+\kappa_{2}\right)\left(\left[\kappa_{b s}\right]^{2}+\left[\kappa_{1}+\operatorname{div} \overrightarrow{\mathbf{e}_{2}}\right]^{2}\right)
$$

An exclusive case is that for which the inextensible soliton surface associated with the Heisenberg antiferromagnetic flow of magnetic $b$-lines is developable. For that, we first assume that magnetic $b$-lines are lines of curvature. Then, the soliton surface is developable if and only if

$$
\left(\kappa_{1}+\operatorname{div} \overrightarrow{\mathbf{e}_{2}}\right) \frac{\partial}{\partial u} \kappa_{b s}-\kappa_{b s} \frac{\partial}{\partial u}\left(\kappa_{1}+\operatorname{div} \overrightarrow{\mathbf{e}_{2}}\right)=\sigma\left(\left[\kappa_{b s}\right]^{2}+\left[\operatorname{div} \overrightarrow{\mathbf{e}_{3}}\right]^{2}\right) .
$$

Equations $(56,57)$ may be used to derive such a relation in a way that emphasizes the physical aspect of the soliton surface through the geometric quantities of the Heisenberg antiferromagnetic flow of magnetic $b$-lines. This relation is written by Laplacian-like differential equations in the following way

$$
\begin{aligned}
-\left(\kappa_{1}+\operatorname{div} \overrightarrow{\mathbf{e}_{2}}\right) \frac{\partial^{2}}{\partial b^{2}} \kappa_{b s}+\kappa_{b s} \frac{\partial^{2}}{\partial b^{2}}\left(\kappa_{1}+\operatorname{div} \overrightarrow{\mathbf{e}_{2}}\right)=\left(\left[\kappa_{b s}\right]^{2}+\left[\kappa_{1}+d i v \overrightarrow{\mathbf{e}_{2}}\right]^{2}\right) \\
\times \frac{\partial}{\partial b}\left(\kappa_{n}+\kappa_{2}\right)+\left(\kappa_{n}+\kappa_{2}\right) \frac{\partial}{\partial b}\left(\left[\kappa_{b s}\right]^{2}+\left[\kappa_{1}+\operatorname{div} \overrightarrow{\mathbf{e}_{2}}\right]^{2}\right], \\
\left(\kappa_{1}+\operatorname{div} \overrightarrow{\mathbf{e}_{2}}\right) \frac{\partial^{2}}{\partial u^{2}} \kappa_{b s}-\kappa_{b s} \frac{\partial^{2}}{\partial u^{2}}\left(\kappa_{1}+\operatorname{div} \overrightarrow{\mathbf{e}_{2}}\right)=\left(\left[\kappa_{b s}\right]^{2}+\left[\kappa_{1}+\operatorname{div} \overrightarrow{\mathbf{e}_{2}}\right]^{2}\right) \frac{\partial}{\partial u} \sigma+\sigma \frac{\partial}{\partial u}\left(\left[\kappa_{b s}\right]^{2}+\left[\kappa_{1}+\operatorname{div} \overrightarrow{\mathbf{e}_{2}}\right]^{2}\right) .
\end{aligned}
$$

In the appendix section, we will investigate approximate solutions and their numerical demonstrations of some special cases of the Laplacian-like formalism given by Eqs. $(58,59)$.

\section{Magnetic Flux Surfaces of the Inextensible Heisenberg Antiferromagnetic Flow}

In this section, new developments of the research recently proposed by many authors on the generalization of time evolution equations on geometric quantities are investigated. Particularly, we consider applications to three-dimensional inextensible Heisenberg antiferromagnetic flow dynamics, including the case of a magnetic flux surface and the flow rotation in the binormal direction. Integrals on the total geometric phase are proved to be related to the integrals, which represent the geometric characterization of the magnetic flux surface in continuous Heisenberg antiferromagnetic spin of magnetic $b$-lines in the binormal direction.

Let us first consider the Lorentz force fields of magnetic $b$-lines given by Eq. (26) .

$$
\phi_{b}\left(\overrightarrow{\mathbf{e}_{1}}\right)=\rho \overrightarrow{\mathbf{e}_{2}}+\kappa_{b s} \overrightarrow{\mathbf{e}_{3}}, \phi_{b}\left(\overrightarrow{\mathbf{e}_{2}}\right)=-\rho \overrightarrow{\mathbf{e}_{1}}+\left(\kappa_{1}+\operatorname{div} \overrightarrow{\mathbf{e}_{2}}\right) \overrightarrow{\mathbf{e}_{3}}, \quad \phi_{b}\left(\overrightarrow{\mathbf{e}_{3}}\right)=-\kappa_{b s} \overrightarrow{\mathbf{e}_{1}}-\left(\kappa_{1}+\operatorname{div} \overrightarrow{\mathbf{e}_{2}}\right) \overrightarrow{\mathbf{e}_{2}}
$$


Now, we are ready to compute the evolution equations of Lorentz force fields for both $b$ and $u$ parameters. The evolution equations of the Lorentz force fields for the arc-length parameter in the binormal direction are written by using Eqs. $(5,60)$ in the following way

$$
\begin{aligned}
\frac{\partial}{\partial b} \phi_{b}\left(\overrightarrow{\mathbf{e}_{1}}\right) & =\left(-\left[\kappa_{b s}\right]^{2}+\rho\left[\kappa_{n}+\kappa_{2}\right]\right) \overrightarrow{\mathbf{e}_{1}}+\left(\frac{\partial}{\partial b} \rho-\kappa_{b s}\left[\kappa_{1}+d i v \overrightarrow{\mathbf{e}_{2}}\right]\right) \overrightarrow{\mathbf{e}_{2}}+\left(\frac{\partial}{\partial b} \kappa_{b s}+\rho\left[\kappa_{1}+d i v \overrightarrow{\mathbf{e}_{2}}\right]\right) \overrightarrow{\mathbf{e}_{3}}, \\
\frac{\partial}{\partial b} \phi_{b}\left(\overrightarrow{\mathbf{e}_{2}}\right) & =\left(-\frac{\partial}{\partial b} \rho-\kappa_{b s}\left[\kappa_{1}+\operatorname{div} \overrightarrow{\mathbf{e}_{2}}\right]\right) \overrightarrow{\mathbf{e}_{1}}+\left(\rho\left[\kappa_{n}+\kappa_{2}\right]-\left[\kappa_{1}+\operatorname{div} \overrightarrow{\mathbf{e}_{2}}\right]^{2}\right) \overrightarrow{\mathbf{e}_{2}}+\left(\frac{\partial}{\partial b}\left[\kappa_{1}+\operatorname{div} \overrightarrow{\mathbf{e}_{2}}\right]-\rho \kappa_{b s}\right) \overrightarrow{\mathbf{e}_{3}} \\
\frac{\partial}{\partial b} \phi_{b}\left(\overrightarrow{\mathbf{e}_{3}}\right) & =\left(-\frac{\partial}{\partial b} \kappa_{b s}-\left[\kappa_{1}+\operatorname{div} \overrightarrow{\mathbf{e}_{2}}\right]\left[\kappa_{n}+\kappa_{2}\right]\right) \overrightarrow{\mathbf{e}_{1}} \\
& +\left(-\frac{\partial}{\partial b}\left[\kappa_{1}+\operatorname{div} \overrightarrow{\mathbf{e}_{2}}\right]+\kappa_{b s}\left[\kappa_{n}+\kappa_{2}\right]\right) \overrightarrow{\mathbf{e}_{2}}-\left(\left[\kappa_{b s}\right]^{2}+\left[\kappa_{1}+d i v \overrightarrow{\mathbf{e}_{2}}\right]^{2}\right) \overrightarrow{\mathbf{e}_{3}}
\end{aligned}
$$

The evolution equations of the Lorentz force fields for the time parameter obtained through the inextensible Heisenberg antiferromagnetic flow are given by using Eqs. $(39-41,60)$ in the following way

$$
\begin{aligned}
\frac{\partial}{\partial u} \phi_{b}\left(\overrightarrow{\mathbf{e}_{1}}\right) & =\left(-\rho \sigma+\kappa_{b s}\left[\frac{\partial}{\partial b}\left\{\kappa_{1}+\operatorname{div} \overrightarrow{\mathbf{e}_{2}}\right\}-\kappa_{b s}\left\{\kappa_{n}+\kappa_{2}\right\}\right]\right) \overrightarrow{\mathbf{e}_{1}}+\left(\frac{\partial}{\partial u} \rho-\kappa_{b s}\left[\frac{\partial}{\partial b} \kappa_{b s}+\left\{\kappa_{1}+\operatorname{div} \overrightarrow{\mathbf{e}_{2}}\right\}\left\{\kappa_{n}+\kappa_{2}\right\}\right]\right) \overrightarrow{\mathbf{e}_{2}} \\
& +\left(\frac{\partial}{\partial u} \kappa_{b s}+\rho\left[\frac{\partial}{\partial b} \kappa_{b s}+\left\{\kappa_{1}+\operatorname{div} \overrightarrow{\mathbf{e}_{2}}\right\}\left\{\kappa_{n}+\kappa_{2}\right\}\right]\right) \overrightarrow{\mathbf{e}_{3}} \\
\frac{\partial}{\partial u} \phi_{b}\left(\overrightarrow{\mathbf{e}_{2}}\right) & =\left(-\frac{\partial}{\partial u} \rho+\left[\kappa_{1}+\operatorname{div} \overrightarrow{\mathbf{e}_{2}}\right]\left[\frac{\partial}{\partial b}\left\{\kappa_{1}+\operatorname{div} \overrightarrow{\mathbf{e}_{2}}\right\}-\kappa_{b s}\left\{\kappa_{n}+\kappa_{2}\right\}\right]\right) \overrightarrow{\mathbf{e}_{1}}-\left(\rho \sigma+\left[\frac{\partial}{\partial b} \kappa_{b s}+\left\{\kappa_{1}+\operatorname{div} \overrightarrow{\mathbf{e}_{2}}\right\}\left\{\kappa_{n}+\kappa_{2}\right\}\right]\right) \overrightarrow{\mathbf{e}_{2}} \\
& +\left(\frac{\partial}{\partial u}\left[\kappa_{1}+\operatorname{div} \overrightarrow{\mathbf{e}_{2}}\right]+\rho\left[\frac{\partial}{\partial b}\left\{\kappa_{1}+\operatorname{div} \overrightarrow{\mathbf{e}_{2}}\right\}-\kappa_{b s}\left\{\kappa_{1}+\operatorname{div} \overrightarrow{\mathbf{e}_{2}}\right\}\right]\right) \overrightarrow{\mathbf{e}_{3}} \\
\frac{\partial}{\partial u} \phi_{b}\left(\overrightarrow{\mathbf{e}_{3}}\right) & =\left(-\frac{\partial}{\partial u} \kappa_{b s}+\sigma\left[\kappa_{1}+\operatorname{div} \overrightarrow{\mathbf{e}_{2}}\right]\right) \overrightarrow{\mathbf{e}_{1}}-\left(\sigma \kappa_{b s}+\frac{\partial}{\partial u}\left[\kappa_{1}+\operatorname{div} \overrightarrow{\mathbf{e}_{2}}\right]\right) \overrightarrow{\mathbf{e}_{2}}+\left(\kappa_{b s}\left[\frac{\partial}{\partial b}\left\{\kappa_{1}+\operatorname{div} \overrightarrow{\mathbf{e}_{2}}\right\}-\kappa_{b s}\left\{\kappa_{n}+\kappa_{2}\right\}\right]\right. \\
& \left.-\left[\kappa_{1}+\operatorname{div} \overrightarrow{\mathbf{e}_{2}}\right]\left[\frac{\partial}{\partial b} \kappa_{b s}+\left\{\kappa_{1}+\operatorname{div} \overrightarrow{\mathbf{e}_{2}}\right\}\left\{\kappa_{n}+\kappa_{2}\right\}\right]\right) \overrightarrow{\mathbf{e}_{3}}
\end{aligned}
$$

Considering Eqs. (61-66) with the general reference frame given by Eq. (5), we can identify the geometric phase of magnetic $b$-lines in which their evolution equations are dependent on both $b$ and $u$ parameters. In this way, we can observe the effect of the rotational flow of magnetic $b$-lines in the binormal direction while they evolve in time through the inextensible Heisenberg antiferromagnetic evolution and Frenet-Serret equations. Accordingly, this phase becomes

$$
\begin{aligned}
& \mathcal{G}_{b}^{\mathcal{P}}=\iint \phi_{b}\left(\overrightarrow{\mathbf{e}_{3}}\right) \cdot\left(\frac{\partial}{\partial b} \phi_{b}\left[\overrightarrow{\mathbf{e}_{3}}\right] \times \frac{\partial}{\partial u} \phi_{b}\left[\overrightarrow{\mathbf{e}_{3}}\right]\right) d b d u=\iint\left(-\kappa_{b s}\left[\left\{-\frac{\partial}{\partial b}\left(\kappa_{1}+d i v \overrightarrow{\mathbf{e}_{2}}\right)+\kappa_{b s}\left(\kappa_{n}+\kappa_{2}\right)\right\}\right.\right. \\
& \times\left\{\kappa_{b s}\left(\frac{\partial}{\partial b}\left\{\kappa_{1}+\operatorname{div} \overrightarrow{\mathbf{e}_{2}}\right\}-\kappa_{b s}\left[\kappa_{n}+\kappa_{2}\right\}\right)-\left(\kappa_{1}+\operatorname{div} \overrightarrow{\mathbf{e}_{2}}\right)\left(\frac{\partial}{\partial b} \kappa_{b s}+\left\{\kappa_{1}+\operatorname{div} \overrightarrow{\mathbf{e}_{2}}\right]\left\{\kappa_{n}+\kappa_{2}\right\}\right)\right\} \\
& \left.-\left\{\left(\kappa_{b s}\right)^{2}+\left(\kappa_{1}+\operatorname{div} \overrightarrow{\mathbf{e}_{2}}\right)^{2}\right\}\left\{\sigma \kappa_{b s}+\frac{\partial}{\partial u}\left(\kappa_{1}+\operatorname{div} \overrightarrow{\mathbf{e}_{2}}\right)\right\}\right]-\left[\kappa_{1}+\operatorname{div} \overrightarrow{\mathbf{e}_{2}}\right]\left[\left\{\frac{\partial}{\partial b} \kappa_{b s}+\left(\kappa_{1}+\operatorname{div} \overrightarrow{\mathbf{e}_{2}}\right)\left(\kappa_{n}+\kappa_{2}\right)\right\}\right. \\
& \times\left\{\kappa_{b s}\left(\frac{\partial}{\partial b}\left\{\kappa_{1}+\operatorname{div} \overrightarrow{\mathbf{e}_{2}}\right\}-\kappa_{b s}\left\{\kappa_{n}+\kappa_{2}\right\}\right)-\left(\kappa_{1}+\operatorname{div} \overrightarrow{\mathbf{e}_{2}}\right)\left(\frac{\partial}{\partial b} \kappa_{b s}+\left\{\kappa_{1}+\operatorname{div} \overrightarrow{\mathbf{e}_{2}}\right\}\left\{\kappa_{n}+\kappa_{2}\right\}\right)\right\} \\
& \left.\left.-\left\{\left(\kappa_{b s}\right)^{2}+\left(\kappa_{1}+\operatorname{div} \overrightarrow{\mathbf{e}_{2}}\right)^{2}\right\}\left\{-\frac{\partial}{\partial u} \kappa_{b s}+\sigma\left(\kappa_{1}+\operatorname{div} \overrightarrow{\mathbf{e}_{2}}\right)\right\}\right]\right) d b d u
\end{aligned}
$$


This is the statement for the geometric phase of the inextensible Heisenberg antiferromagnetic flow of magnetic $b$-lines in the binormal direction. We use an efficient technique that is based on the certain evolution system of the spin configuration, which allows the system to return to its original shape and position after a time interval. This technique also provides to determine necessary and sufficient conditions that have to be satisfied by the geometric quantities associated with the magnetic $b$-lines to define the Lorentz magnetic flux surfaces. Finally, it can be considered to compute the magnetic flux density of the Lorentz magnetic flux surfaces obtained through the evolution of magnetic $b$-lines based on the inextensible Heisenberg antiferromagnetic flow model.

The magnetic vector field of magnetic $b$-lines in the binormal direction has already computed by Eq. (28) in the following way

$$
\mathcal{M}_{b}=\left(\kappa_{1}+\operatorname{div} \overrightarrow{\mathbf{e}_{2}}\right) \overrightarrow{\mathbf{e}_{1}}-\kappa_{b s} \overrightarrow{\mathbf{e}_{2}}+\rho \overrightarrow{\mathbf{e}_{3}}
$$

Using the Lorentz force equations and inextensible Heisenberg antiferromagnetic evolution equations of magnetic $b$-lines with appropriate boundary conditions given by Eqs. $(60-66)$, the necessary and sufficient conditions for the existence of the Lorentz magnetic flux surfaces are stated by

$$
\begin{aligned}
0 & =\left(\kappa_{1}+\operatorname{div} \overrightarrow{\mathbf{e}_{2}}\right)\left([ - \frac { \partial } { \partial b } \{ \kappa _ { 1 } + \operatorname { d i v } \vec { \mathbf { e } _ { 2 } } \} + \kappa _ { b s } \{ \kappa _ { n } + \kappa _ { 2 } \} ] \left[\kappa_{b s}\left\{\frac{\partial}{\partial b}\left(\kappa_{1}+\operatorname{div} \overrightarrow{\mathbf{e}_{2}}\right)-\kappa_{b s}\left(\kappa_{n}+\kappa_{2}\right)\right\}-\left\{\kappa_{1}+\operatorname{div} \overrightarrow{\mathbf{e}_{2}}\right\}\right.\right. \\
& \left.\left.\times\left\{\frac{\partial}{\partial b} \kappa_{b s}+\left(\kappa_{1}+\operatorname{div} \overrightarrow{\mathbf{e}_{2}}\right)\left(\kappa_{n}+\kappa_{2}\right)\right\}\right]-\left[\left\{\kappa_{b s}\right\}^{2}+\left\{\kappa_{1}+\operatorname{div} \overrightarrow{\mathbf{e}_{2}}\right\}^{2}\right]\left[\sigma \kappa_{b s}+\frac{\partial}{\partial u}\left\{\kappa_{1}+\operatorname{div} \overrightarrow{\mathbf{e}_{2}}\right\}\right]\right) \\
& -\kappa_{b s}\left([ \frac { \partial } { \partial b } \kappa _ { b s } + \{ \kappa _ { 1 } + \operatorname { d i v } \vec { \mathbf { e } _ { 2 } } \} \{ \kappa _ { n } + \kappa _ { 2 } \} ] \left[\kappa_{b s}\left\{\frac{\partial}{\partial b}\left(\kappa_{1}+\operatorname{div} \overrightarrow{\mathbf{e}_{2}}\right)-\kappa_{b s}\left(\kappa_{n}+\kappa_{2}\right)\right\}-\left\{\kappa_{1}+\operatorname{div} \overrightarrow{\mathbf{e}_{2}}\right\}\right.\right. \\
& \left.\left.\times\left\{\frac{\partial}{\partial b} \kappa_{b s}+\left(\kappa_{1}+\operatorname{div} \overrightarrow{\mathbf{e}_{2}}\right)\left(\kappa_{n}+\kappa_{2}\right)\right\}\right]-\left[\left\{\kappa_{b s}\right\}^{2}+\left\{\kappa_{1}+\operatorname{div} \overrightarrow{\mathbf{e}_{2}}\right\}^{2}\right]\left[-\frac{\partial}{\partial u} \kappa_{b s}+\sigma\left\{\kappa_{1}+\operatorname{div} \overrightarrow{\mathbf{e}_{2}}\right\}\right]\right) \\
& +\rho\left(\left[\frac{\partial}{\partial b} \kappa_{b s}+\left\{\kappa_{1}+\operatorname{div} \overrightarrow{\mathbf{e}_{2}}\right\}\left\{\kappa_{n}+\kappa_{2}\right\}\right]\left[\sigma \kappa_{b s}+\frac{\partial}{\partial u}\left\{\kappa_{1}+\operatorname{div} \overrightarrow{\mathbf{e}_{2}}\right\}\right]+\left[\frac{\partial}{\partial b}\left\{\kappa_{1}+\operatorname{div} \overrightarrow{\mathbf{e}_{2}}\right\}-\kappa_{b s}\left\{\kappa_{n}+\kappa_{2}\right\}\right]\right. \\
& \left.\times\left[-\frac{\partial}{\partial u} \kappa_{b s}+\sigma\left\{\kappa_{1}+\operatorname{div} \overrightarrow{\mathbf{e}_{2}}\right\}\right]\right) .
\end{aligned}
$$

As a result, the magnetic flux density of the Lorentz magnetic flux surfaces obtained through the evolution of magnetic $b$-lines based on the inextensible Heisenberg antiferromagnetic flow model is computed by

$$
\begin{aligned}
& \mathcal{F}_{b}^{\mathcal{D}}=\iint\left([ \kappa _ { 1 } + \operatorname { d i v } \vec { \mathbf { e } _ { 2 } } ] \left[\{ - \frac { \partial } { \partial b } ( \kappa _ { 1 } + \operatorname { d i v } \vec { \mathbf { e } _ { 2 } } ) + \kappa _ { b s } ( \kappa _ { n } + \kappa _ { 2 } ) \} \left\{\kappa_{b s}\left(\frac{\partial}{\partial b}\left[\kappa_{1}+\operatorname{div} \overrightarrow{\mathbf{e}_{2}}\right]-\kappa_{b s}\left[\kappa_{n}+\kappa_{2}\right]\right)\right.\right.\right. \\
& \left.\left.-\left(\kappa_{1}+\operatorname{div} \overrightarrow{\mathbf{e}_{2}}\right)\left(\frac{\partial}{\partial b} \kappa_{b s}+\left[\kappa_{1}+\operatorname{div} \overrightarrow{\mathbf{e}_{2}}\right]=\left[\kappa_{n}+\kappa_{2}\right]\right)\right\}-\left\{\left(\kappa_{b s}\right)^{2}+\left(\kappa_{1}+\operatorname{div} \overrightarrow{\mathbf{e}_{2}}\right)^{2}\right\}\left\{\sigma \kappa_{b s}+\frac{\partial}{\partial u}\left(\kappa_{1}+d i v \overrightarrow{\mathbf{e}_{2}}\right)\right\}\right] \\
& -\kappa_{b s}\left[\{ \frac { \partial } { \partial b } \kappa _ { b s } + ( \kappa _ { 1 } + \operatorname { d i v } \vec { \mathbf { e } _ { 2 } } ) ( \kappa _ { n } + \kappa _ { 2 } ) \} \left\{\kappa_{b s}\left(\frac{\partial}{\partial b}\left[\kappa_{1}+\operatorname{div} \overrightarrow{\mathbf{e}_{2}}\right]-\kappa_{b s}\left[\kappa_{n}+\kappa_{2}\right]\right)-\left(i \kappa_{1}+\operatorname{div} \overrightarrow{\mathbf{e}_{2}}\right)\left(\frac{\partial}{\partial b} \kappa_{b s}\right.\right.\right. \\
& \left.\left.+\left\{\kappa_{1}+\operatorname{div} \overrightarrow{\mathbf{e}_{2}}\right]-\left\{\kappa_{n}+\kappa_{2}\right]\right)\right\}-\left\{\left(\kappa_{b s}\right)^{2}+\left(\kappa_{1}+\operatorname{div} \overrightarrow{\mathbf{e}_{2}}\right)^{2}\right\}\left\{-\frac{\partial}{\partial u} \kappa_{b s}+\sigma\left(\kappa_{1}+\operatorname{div} \overrightarrow{\mathbf{e}_{2}}\right)\right\}+\rho\left\{\left(\frac{\partial}{\partial b} \kappa_{b s}\right.\right. \\
& \left.+=\left[\kappa_{1}+\operatorname{div} \overrightarrow{\mathbf{e}_{2}}\right]=\left[\kappa_{n}+\kappa_{2}\right]\right)\left(\sigma \kappa_{b s}+\frac{\partial}{\partial u}\left[\kappa_{1}+\operatorname{div} \overrightarrow{\mathbf{e}_{2}}\right]\right)+\left(\frac{\partial}{\partial b}\left[\kappa_{1}+\operatorname{div} \overrightarrow{\mathbf{e}_{2}}\right]-\kappa_{b s}\left[\kappa_{n}+\kappa_{2}\right]\right) \\
& \left.\left.\left.\times\left(-\frac{\partial}{\partial u} \kappa_{b s}+\sigma\left\{\kappa_{1}+\operatorname{div} \overrightarrow{\mathbf{e}_{2}}\right\}\right)\right\}\right]\right) d b d u
\end{aligned}
$$




\section{Fractional solutions of Laplacian-like equa- tions with conformable fractional derivative}

In this section, the connection between the Laplacian-like non-linear equation the celebrated inextensible Heisenberg antiferromagnetic flow is investigated in the binormal direction. In Eqs. (58,59), we have already induced solitonic equations that are associated with curves of geometric quantities. If one considers the appropriate limiting and scaling process, then a basic geometric derivation admits the following reciprocal transformation

$$
\begin{gathered}
\left(\kappa_{1}+\operatorname{div} \overrightarrow{\mathbf{e}_{2}}\right) \frac{\partial^{2}}{\partial b^{2}} \kappa_{b s}-\kappa_{b s} \frac{\partial^{2}}{\partial b^{2}}\left(\kappa_{1}+\operatorname{div} \overrightarrow{\mathbf{e}_{2}}\right)-\frac{\partial^{\alpha}}{\partial u^{\alpha}} \kappa_{b s}=0 \\
\left(\kappa_{1}+\operatorname{div} \overrightarrow{\mathbf{e}_{2}}\right) \frac{\partial^{2 \alpha}}{\partial u^{2 \alpha}} \kappa_{b s}-\kappa_{b s} \frac{\partial^{2 \alpha}}{\partial u^{2 \alpha}}\left(\kappa_{1}+\operatorname{div} \overrightarrow{\mathbf{e}_{2}}\right) \\
-\frac{\partial}{\partial b}\left(\kappa_{1}+\operatorname{div} \overrightarrow{\mathbf{e}_{2}}\right)=0
\end{gathered}
$$

where $\partial^{\alpha} / \partial u^{\alpha}$ is the conformable derivative operator. These fractional equations reflect the propagation of solitonic surface sweeping out as geometric quantities evolve for time invariance in the binormal direction. A lot of research has been done using different fractional operators for fractional differential equations [42-44].

The conformable derivative of order $\eta \in(0,1]$ is defined by the following equation [45]

$$
\begin{aligned}
{ }_{t} D^{\eta} f(t) & =\lim _{\vartheta \rightarrow 0} \frac{f\left(t+\vartheta t^{1-\eta}\right)-f(t)}{\vartheta}, \\
f & :(0, \infty) \rightarrow \mathbb{R} .
\end{aligned}
$$

Some of the features of the conformable derivative are given as follows [45-47]

$$
\begin{aligned}
\text { a) }{ }_{t} D^{\eta} t^{\alpha} & =\alpha t^{\alpha-\eta}, \quad \forall \eta \in \mathbb{R}, \\
\text { b) }{ }_{t} D^{\eta}(f g) & =f_{t} D^{\eta} g+g_{t} D^{\eta} f, \\
c) \quad{ }_{t} D^{\eta}(f o g) & =t^{1-\eta} g^{\prime}(t) f^{\prime}(g(t)), \\
\text { d) }{ }_{t} D^{\eta}\left(\frac{f}{g}\right) & =\frac{g_{t} D^{\eta} f-f_{t} D^{\eta} g}{g^{2}} .
\end{aligned}
$$

We consider the given below traveling wave transformation for Eqs. (69)

$$
\begin{aligned}
\kappa_{b s} & =r(\phi), \\
\kappa_{1}+\operatorname{div} \overrightarrow{\mathbf{e}_{2}} & =v(\phi), \quad \phi=n-Q \frac{u^{\alpha}}{\alpha}
\end{aligned}
$$

where $Q$ describes the speed of the wave. If one places Eq. (71) into Eq. (69) and considers the imaginary section, then it is obtained that

$$
\begin{aligned}
Q r^{\prime}(\phi)+v(\phi) r^{\prime \prime}(\phi)-r(\phi) v^{\prime \prime}(\phi) & =0 \\
Q^{2} v(\phi) r^{\prime \prime}(\phi)-v^{\prime}(\phi)-Q^{2} r(\phi) v^{\prime \prime}(\phi) & =0 .
\end{aligned}
$$

Solutions of Eq. (72) can be written as a series expansion in the following way

$$
\begin{aligned}
& r(\phi)=\alpha_{0}+\alpha_{1} G(\phi)+\alpha_{2} G^{-1}(\phi), \\
& v(\phi)=\beta_{0}+\beta_{1} G(\phi)+\beta_{2} G^{-1}(\phi),
\end{aligned}
$$

where $\alpha_{0}, \alpha_{1}, \alpha_{2}, \beta_{0}, \beta_{1}, \beta_{2}$ are functions to be determined later, and $G(\phi)$ satisfies the following fractional Riccati equation

$$
G^{\prime}(\phi)=\sigma+G^{2}(\phi),
$$

where $\sigma$ is an arbitrary constant.

- $\quad N$ is obtained with the aid of balance between the highest order derivatives and the nonlinear terms in Eq. (72).

A few special solutions of Eq. (74) are listed in the following manner.

1) When $\sigma<0$,

$$
\begin{aligned}
& G_{1}(\phi)=-\sqrt{-\sigma} \tanh (\sqrt{-\sigma} \phi), \\
& G_{2}(\phi)=-\sqrt{-\sigma} \operatorname{coth}(\sqrt{-\sigma} \phi) .
\end{aligned}
$$

2) When $\sigma>0$,

$$
\begin{aligned}
& G_{3}(\phi)=\sqrt{\sigma} \tan (\sqrt{\sigma} \phi), \\
& G_{4}(\phi)=\sqrt{\sigma} \cot (\sqrt{\sigma} \phi) .
\end{aligned}
$$

3) When $\sigma=0, \rho=$ const.,

$$
G_{5}(\phi)=-\frac{1}{\phi+\rho} .
$$

Now, if one replaces Eqs. (73) and (74) into Eq. (72), and equated all coefficients of $G(\phi)$, then it is obtained some special functions as follows

$$
\beta_{0}=-Q^{2} \alpha_{0}, \beta_{1}=-\frac{Q^{4} \alpha_{1}}{2 \sigma}, \beta_{2}=-Q^{3} \alpha_{2} .
$$

Furthermore, if one uses the fact that $\sigma=-1, G(\phi)=$ $-\sqrt{-\sigma} \operatorname{coth}(\sqrt{-\sigma} \phi)$, then it is computed that

$$
\begin{aligned}
r(\phi) & =\alpha_{0}-\alpha_{1} \sqrt{-\sigma} \operatorname{coth}(\sqrt{-\sigma} \phi) \\
& -\alpha_{2}(\sqrt{-\sigma} \operatorname{coth}[\sqrt{-\sigma} \phi])^{-1}, \\
v(\phi) & =-Q^{2} \alpha_{0}+\frac{Q^{4} \alpha_{1}}{2 \sigma} \sqrt{-\sigma} \operatorname{coth}(\sqrt{-\sigma} \phi) \\
& +Q^{3} \alpha_{2}(\sqrt{-\sigma} \operatorname{coth}[\sqrt{-\sigma} \phi])^{-1} .
\end{aligned}
$$

As a result, one gets the following solution family for Eqs. (69)

$$
\begin{aligned}
\kappa_{b s} & =\alpha_{0}-\alpha_{1} \sqrt{-\sigma} \operatorname{coth}(\sqrt{-\sigma} \phi) \\
& -\alpha_{2}(\sqrt{-\sigma} \operatorname{coth}[\sqrt{-\sigma} \phi])^{-1} \\
\kappa_{1}+\operatorname{div} \overrightarrow{\mathbf{e}_{2}} & =-Q^{2} \alpha_{0}+\frac{Q^{4} \alpha_{1}}{2 \sigma} \sqrt{-\sigma} \operatorname{coth}(\sqrt{-\sigma} \phi) \\
& +Q^{3} \alpha_{2}(\sqrt{-\sigma} \operatorname{coth}[\sqrt{-\sigma} \phi])^{-1} .
\end{aligned}
$$



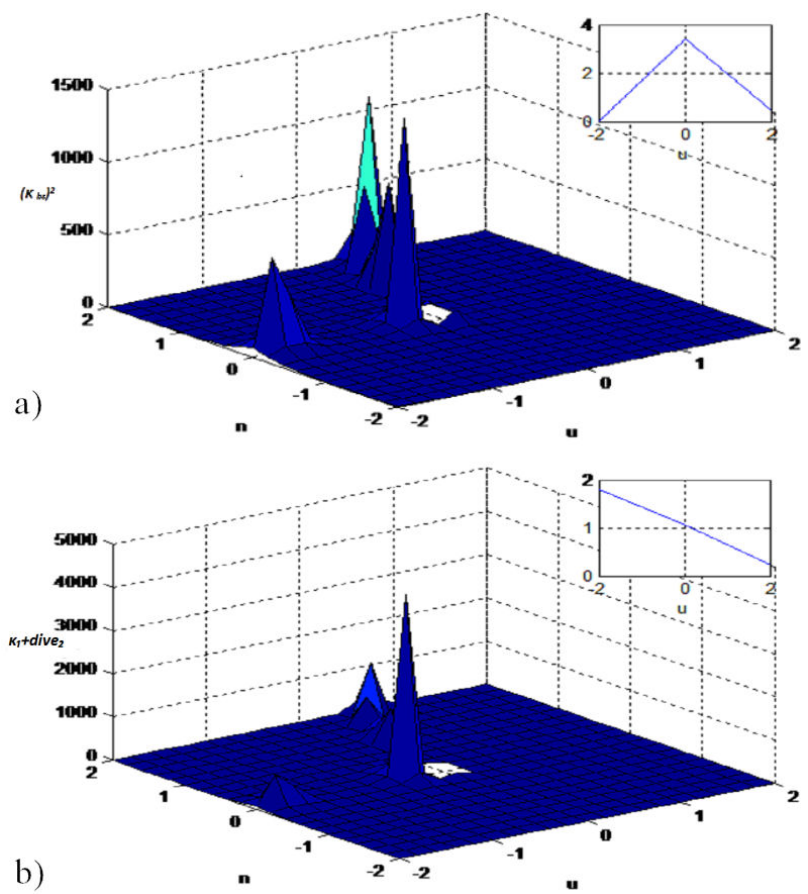

FIGURE 1. The 3D graphic for analytical solutions of the fractional equations.

Figure 1 is the 3D graphic for analytical solutions of the fractional Eq. (69) for $\alpha=0.5, Q=1.2, \alpha_{0}=0, \alpha_{1}=$ $-2, \alpha_{2}=2$. a) for $\kappa_{b s}$ in solutions Eq. (78) b) for $\kappa_{1}+\operatorname{div} \overrightarrow{\mathbf{e}_{2}}$ in solutions Eq. (79).

\section{Conclusion}

One of the most important properties of the electromagnetic vector field at the Riemannian geometry is its degree of spatial intermittency. Magnetic flux surface mostly occurs in the shape of isolated, intense elements encompassed by nearly flux-free or flux-free structures. According to conceptual appeal and its basic geometrical construction, we have developed a simpler approach to define magnetic phenomena. Accordingly, this approach is reconciled with the more principal definition of electromagnetic components in terms of smooth non-vanishing fields by supposing that the inextensible Heisenberg antiferromagnetic evolution of magnetic $b$ lines creates a Lorentz magnetic flux surface. The fundamental constructing blocks for our argument are to define magnetic $b$-lines through the Lorentz force and the geometric quantities of the Frenet-Serret frame. In this way, this study may lead to modeling a more geometrical and simplified version of the magnetic flux tube soon. Another objective may be investigating the effect of other magnetic lines and their geometries in a system where their evolutions form magnetic flux surfaces or magnetic flux tubes. This analysis may also be expanded by considering different kinds of flow models apart from inextensible Heisenberg antiferromagnetic flow.

The results demonstrate that the considered method is more effective and easy to employ to scrutinize the behaviors of the fractional differential equations with magnetic flux surfaces or magnetic flux tubes arising in associated areas of science and technology. Additionally, we obtain the 3D graphic for analytical solutions of the Laplacian-like non-linear equation.

All in all, this study provides a unique insight for defining magnetic flux surfaces through the intense consideration of differential geometric tools. However, the potential physical effects of this research may be seen in Hermitian and non-Hermitian wave physics, topological quantum states, and Maxwell electromagnetism immediately. Moreover, investigating its implication in practical fields such as metamaterials or magnetic materials will be the final and decisive goal to complete the paper in all senses.
1. R. Gilmore, Length and curvature in the geometry of thermodynamics, Physical Review A 30 (1984) 1994, https: //doi.org/10.1103/PhysRevA.30.1994

2. B. M. Barbashov and V. Nesterenko, Introduction to the relativistic string theory (World Scientific, 1990).

3. V. De Sabbata, C. Sivaram, Spin and torsion in gravitation (World Scientific, 1994).

4. W. K. Schief and C. Rogers, The Da Rios system under a geometric constraint: the Gilbarg problem, Journal of Geometry and Physics 54 (2005) 286, https://doi.org/10. $1016 / j$.geomphys.2004.10.001.

5. R. G. Littlejohn, Variational principles of guiding centre motion, Journal of Plasma Physics 29 (1983) 111, https:// doi.org/10.1017/S002237780000060X.

6. M. Kleman, Developable domains in hexagonal liquid crystals, Journal de Physique 41 (1980) 737, https://doi.org/ 10.1051/jphys:01980004107073700.
7. T. Körpinar and R. C. Demirkol, Electromagnetic curves of the linearly polarized light wave along an optical fiber in a 3D Riemannian manifold with Bishop equations, $O p$ tik 200 (2020) 163334, https://doi.org/10.1080/ 09500340.2019 .1579930 .

8. T. Körpinar and R. C. Demirkol, Frictional magnetic curves in 3D Riemannian manifolds, International Journal of Geometric Methods in Modern Physics 15 (2018) 1850020, https: //doi.org/10.1142/S0219887818500202

9. T. Körpinar and R. C. Demirkol, Gravitational magnetic curves on 3D Riemannian manifolds, International Journal of Geometric Methods in Modern Physics, 15 (2018) 1850184, https: //doi.org/10.1142/S0219887818501840.

10. A. Kazan and H. B. Karadag, Magnetic pseudo null and magnetic null curves in Minkowski 3-space, International Mathematical Forum 123 (2017) 119 https://doi.org/10. $12988 / \mathrm{imf} .2017 .612168$. 
11. Ş. Güvenç and C. Özgür, On slant magnetic curves in Smanifolds, Journal of Nonlinear Mathematical Physics 26 (2019) 536, https://doi.org/10.1080/14029251. 2019.1640463 .

12. J. L. Cabrerizo, Magnetic fields in 2D and 3D sphere, Journal of Nonlinear Mathematical Physics 20 (2013) 440, https: //doi.org/10.1080/14029251.2013.855052.

13. J. Sun, Singularity properties of killing magnetic curves in Minkowski 3-space, International Journal of Geometric Methods in Modern Physics 16 (2019) 1950123, https : / / doi. org/10.1142/S0219887820501418.

14. T. Körpınar, R. C. Demirkol, Z. Körpınar and V. Asil, Maxwellian evolution equations along the uniform optical fiber in Minkowski space, Revista Mexicana de Física 66 (2020) 431, https://doi.org/10.31349/RevMexFis.66. 431.

15. T. Körpınar, R. C. Demirkol, Z. Körpınar and V. Asil, Maxwellian evolution equations along the uniform optical fiber, Optik 217 (2020) 164561, https: //doi.org/10.1016/ j.ijleo.2020.164561.

16. R. L. Ricca, Inflexional disequilibrium of magnetic flux-tubes, Fluid Dynamics Research 36 (2005) 319, https://doi. org/10.1016/j.fluiddyn.2004.09.004.

17. R. L. Ricca, Evolution and inflexional instability of twisted magnetic flux tubes, Solar Physics 172 (1997) 241 https: //doi.org/10.1023/A:1004942121307.

18. L. C. Garcia de Andrade, Non-Riemannian geometry of twisted flux tubes, Brazilian Journal of Physics 36 (2006) 1290, https://doi.org/10.1590/ s0103-97332006000700030.

19. L. C. Garcia de Andrade, Riemannian geometry of twisted magnetic flux tubes in almost helical plasma flows, Physics of Plasmas 13 (2006) 022309, https://doi.org/10. $1063 / 1.2172363$.

20. L. C. Garcia de Andrade, Vortex filaments in MHD, Physica Scripta 73 (2006) 484, https : / / doi .org/10.1088/ 0031-8949/73/5/012.

21. B. Guo and S. Ding, Landau-Lifshitz Equations (World Scientific, 2008).

22. V. R. Vieira and P. P. Horley, The Frenet-Serret representation of the Landau-Lifshitz-Gilbert equation, Journal of Physics A: Mathematical and Theoretical 45 (2012) 065208, https: //doi.org/10.1088/1751-8113/45/6/065208

23. H. Hasimoto, A soliton on a vortex filament, Journal of Fluid Mechanics 51 (1972) 477, https: //doi.org/10.1017/ S0022112072002307.

24. S. C. Anco and R. Myrzakulov, Integrable generalizations of Schrödinger maps and Heisenberg spin models from Hamiltonian flows of curves and surfaces, Journal of Geometry and Physics 60 (2010) 1576.

25. M. Erdoğdu and M. Özdemir, Geometry of Hasimoto surfaces in Minkowski 3-space, Mathematical Physics, Analysis and Geometry 17 (2014) 169, https://doi.org/10.1007/ s11040-014-9148-3.
26. R. L. Ricca, Physical interpretation of certain invariants for vortex filament motion under LIA, Physics of Fluids A: Fluid Dynamics 4 (1992) 938, https://doi.org/10.1063/1. 858274 .

27. R. Balakrishnan, A. R. Bishop and R. Dandoloff, Anholonomy of a moving space curve and applications to classical magnetic chains, Physical Review B 47 (1993) 3108, https: //doi.org/10.1103/PhysRevB.47.3108.

28. M. Barros, A. Ferrández, P. Lucas and M. Merono, Hopf cylinders, B-scrolls and solitons of the Betchov-Da Rios equation in the 3-dimensional anti-De Sitter space, CR Acad. Sci. Paris, Série I, 321 (1995) 505.

29. M. Barros, A. Ferrández, P. Lucas, and M. A. Meroño, Solutions of the Betchov-Da Rios soliton equation: a Lorentzian approach, Journal of Geometry and Physics 31 (1999) 217, https://doi.org/10.1016/S0393-0440(99) $00005-4$.

30. J. Arroyo, O. Garay, A. Pámpano, Binormal motion of curves with constant torsion in 3-spaces, Advances in Mathematical Physics (2017) 2017, https://doi.org/10.1155/ $2017 / 7075831$.

31. T. Körpınar, R. C. Demirkol, and Z. Körpınar, Soliton propagation of electromagnetic field vectors of polarized light ray traveling along with coiled optical fiber on the unit 2-sphere $\mathbb{S}^{2}$, Rev. Mex. Fis. 65 (2019) 626 https://doi.org/10. 31349 /revmexfis.65.626.

32. T. Körpınar, R. C. Demirkol, and Z. Körpınar, Soliton propagation of electromagnetic field vectors of polarized light ray traveling in a coiled optical fiber in Minkowski space with Bishop equations, The European Physical Journal D 73 (2019) 203, https://doi.org/10.1140/epjd/ e2019-100250-7.

33. T. Körpınar, R. C. Demirkol, and Z. Körpınar, Soliton propagation of electromagnetic field vectors of polarized light ray traveling in a coiled optical fiber in the ordinary space, International Journal of Geometric Methods in Modern Physics 16 (2019) 1950117, https://doi.org/10. $1142 /$ S0219887819501172.

34. R. Balakrishnan, A. R. Bishop and R. Dandoloff, Geometric phase in the classical continuous antiferromagnetic Heisenberg spin chain, Physical review letters 64 (1990) 2107.

35. K. Y. Bliokh, Geometrodynamics of polarized light: Berry phase and spin Hall effect in a gradient-index medium, Journal of Optics A: Pure and Applied Optics 11 (2009) 094009, https://doi.org/10.1088/1464-4258/11/9/ 094009 .

36. K. Y. Bliokh, A. Niv, V. Kleiner and E. Hasman, Geometrodynamics of spinning light. Nature Photonics 2 (2008) 748, https://doi.org/10.1038/nphoton.2008.229.

37. F. Wassmann and A. Ankiewicz, Berry's phase analysis of polarization rotation in helicoidal fibers, Applied optics, 37 (1998) 3902-39, https://doi.org/10.1364/AO.37. 003902 .

38. R. Balakrishnan, Space curve evolution, geometric phase, and solitons, Theoretical and Mathematical Physics 99 (1994) 501, https://doi.org/10.1007/BF01016130. 
39. J. Samuel and R. Nityananda, Transport along null curves, Journal of Physics A: Mathematical and General 33 (2000) 2895, https://doi.org/10.1088/0305-4470/33/ $14 / 318$.

40. R. Balakrishnan and R. Dandoloff, Classical analogues of the Schrödinger and Heisenberg pictures in quantum mechanics using the Frenet frame of a space curve: an example, European journal of physics, 25 (2004) 447, https : / / doi . org/10. 1088/0143-0807/25/3/012.

41. T. Körpınar, R. C. Demirkol and V. Asil, Directional magnetic and electric vortex lines and their geometries, Indian Journal of Physics (2020), https: //doi .org/10.1007/ s12648-020-01885-2.

42. W. Gao, P. Veeresha, D. G. Prakasha and H. M. Baskonus, Novel dynamical structures of 2019-nCoV with nonlocal operator via powerful computational technique, Biology, 9 (2020) 107, https://doi.org/10.3390/ biology 9050107 .

43. W. Gao, P. Veeresha, D. G. Prakasha, H. M. Baskonus and G. Yel, New Numerical Results for the Time-Fractional PhiFour Equation Using a Novel Analytical Approach, Sym- metry 12 (2020) 478, https://doi.org/10.3390/ sym12030478.

44. W. Gao, P. Veeresha, D. G. Prakasha, H. M. Baskonus, New numerical simulation for fractional Benney-Lin equation arising in falling film problems using two novel techniques, Numerical Methods for Partial Differential Equations, 37 (2021) 210 , https://doi.org/10.1002/num.22526.

45. R. Khalil, M. Al Horani, A. Yousef, and M. Sababheh, A new definition of fractional derivative, Journal of Computational and Applied Mathematics 264 (2014) 65, https://doi. org/10.1016/j.cam.2014.01.002.

46. M. Eslami, H. Rezazadeh, The first integral method for $\mathrm{Wu}-$ Zhang system with conformable time-fractional derivative, Calcolo 53 (2016) 475, https://doi.org/10.1007/ s10092-015-0158-8.

47. Y. Çenesiz, D. Baleanu, A. Kurt and O. Tasbozan, New exact solutions of Burgers' type equations with conformable derivative, Waves in Random and complex Media 27 (2017) 103, https://doi.org/10.1080/17455030.2016. 1205237. 